\title{
Simultaneous single-pulse observations of radio pulsars
}

\section{The behaviour of circular polarization}

\author{
A. Karastergiou ${ }^{1,2}$, S. Johnston ${ }^{2}$, and M. Kramer ${ }^{3}$ \\ 1 Max-Planck Institut für Radioastronomie, Auf dem Hügel 69, 53121 Bonn, Germany \\ 2 School of Physics, University of Sydney, NSW 2006, Australia \\ 3 Jodrell Bank Observatory, University of Manchester, Macclesfield, Chesire SK11 9DL, UK
}

Received 24 December 2002 / Accepted 18 March 2003

\begin{abstract}
We investigate circular polarization in pulsar radio emission through simultaneous observations of PSR B1133+16 at two frequencies. In particular, we investigate the association of the handedness of circular polarization with the orthogonal polarization mode phenomenon at two different frequencies. We find the association to be significant across the pulse for PSR B1133+16, making a strong case for orthogonal polarization modes determining the observed circular polarization. The association however is not perfect and decreases with frequency. Based on these results and assuming emission occurs in superposed orthogonal polarization modes, we present a technique of mode decomposition based on single pulses. Average profiles of the polarization of each mode can then be computed by adding the individual mode-separated single pulses. We show that decomposing single pulses produces different average profiles for the orthogonal polarization modes from decomposing average profiles. Finally, we show sample single pulses and discuss the implications of the frequency dependence of the correlation of the circular polarization with the orthogonal polarization mode phenomenon.
\end{abstract}

Key words. pulsars: individual: PSR B1133+16 - polarization

\section{Introduction}

Perhaps one of the most elusive qualities of the highly polarized pulsar radio emission is its circular polarization. Although it is generally lower than its linearly polarized counterpart, it is amongst the highest observed in astrophysical objects. Circular polarization in pulsars has been attributed in the past to propagation effects on the radiation as it travels through the pulsar magnetosphere, geometrical effects or the emission mechanism itself.

Cordes et al. (1978) were the first to point out an association between the position angle (PA) of the linear polarization and the handedness of the circular polarization. More specifically, $90^{\circ}$ jumps in the PA were observed to occur simultaneously with sense reversals in the circular polarization $(V)$ in PSR B2020+28. The authors argued that this could be adequately explained by disjoint or superposed orthogonal polarization modes (OPMs). In the disjoint case, each of the OPMs is emitted independently and only individual modes are observed at a time. On the other hand, the superposed case supports the idea that the observed radiation at any instant is a mix of both modes, which are effectively emitted at the same time, despite each mode having its own properties. A number of authors argued for the identification of OPMs as the natural

Send offprint requests to: A. Karastergiou, e-mail: aris@physics.usy.edu.au polarization modes in the pulsar magnetosphere (e.g. Barnard \& Arons 1986; McKinnon 1997; von Hoensbroech et al. 1998; Petrova 2001). Elliptically polarized natural modes could then provide the origin for the circular polarization as a propagation effect.

Stinebring et al. (1984a,b) studied the polarization behaviour of single pulses from a number of pulsars using greyscale density plots to investigate the fluctuations of the PA, the linear polarization, $L$ and $V$. They noticed that PA jumps often occur at the same pulse longitude at 430 and $1404 \mathrm{MHz}$ and that the orthogonal polarization mode (OPM) phenomenon was becoming more prevalent at higher frequencies. They also noticed that at the phase bins where OPM jumps occur, the degree of linear polarization was significantly reduced, which led to the idea that, at any given instant, the radiation observed is the incoherent sum of OPMs. This has the advantage of naturally reducing the linear polarization and attributing its fluctuations to fluctuations in the polarization of the OPMs, which have a constant degree of linear polarization. In contrast, if OPMs were emitted independently, the emission mechanism would have to intrinsically produce a largely fluctuating degree of linear polarization. McKinnon \& Stinebring (1998) realized that the assumption of incoherent addition of OPMs can be used to decompose an integrated pulse profile into the profiles of each OPM. By accounting for the effects of instrumental noise, this method can be used to reproduce the histograms of the 
distributions of the PA and $L$ from Stinebring et al. (1984b). Mode decomposed average profiles of PSRs B0525+21 and B2020+28 were shown in McKinnon \& Stinebring (2000) using their technique on the average profiles of these pulsars.

In this paper, the association of circular polarization with the orthogonal polarization modes is examined in PSR B1133+16 at $1.41 \mathrm{GHz}$ and $4.85 \mathrm{GHz}$, having studied the correlation of OPMs between these two frequencies in Karastergiou et al. (2002, hereafter Paper II). We make use of contingency tables to quantify this association at each frequency, which we compare to the findings of Cordes et al. (1978). We develop a method to decompose single pulses into the individual OPMs. The method is simple and only assumes that the observed Stokes $I, Q, U, V$ are the incoherent sum of the $100 \%$ polarized OPMs. We show the different mode-decomposed profiles of PSR B1133+16 by following our method and that of McKinnon \& Stinebring (2000) and discuss the differences. Finally, we present example single-pulses from our simultaneous data set to highlight the observed effects and we discuss possible interpretations.

\section{The data}

We use the same dataset on PSR B1133+16 as in Paper II. It consists of 4778 pulses at 4.85 and $1.41 \mathrm{GHz}$ observed with the Effelsberg and Jodrell Bank radio telescopes respectively. Full details of the observing, calibration and data reduction can be found in Paper II. The mean signal-to-noise ratio $(S / N)$ of $V$ detected in the single pulses is $\ll 1$. However, in a significant number of cases, the signal in $V$ exceeds the rms of the noise by many times at both frequencies. At $4.85 \mathrm{GHz}$, where the flux density of this pulsar is much less than $1.41 \mathrm{GHz}$, the sensitivity of the Effelsberg telescope largely compensates. This is evident from the $S / N$ of $V$ shown in Fig. 1 and favours our results to be intrinsic to the pulsar emission rather than products of the instrumental noise.

\subsection{Distributions of $V$ and $V / I$}

To investigate the single-pulse behaviour of circular polarization, the value of $V$ is plotted against the corresponding pulse phase for every single pulse. Such density plots reveal the pulse to pulse fluctuations of $V$. Figure 1 shows two such plots for the single-pulse data of PSR B1133+16 observed simultaneously at $4.85 \mathrm{GHz}$ and $1.41 \mathrm{GHz}$. The density plots reveal that the distributions of $V$ at each pulse phase bin range over a few tens of rms units at both frequencies.

In the leading component, it has been shown that the OPMs are almost equally strong and dominate equally often at both frequencies (Paper II). The distribution of $V$ in this component shows an almost equally dense population of positive and negative values. On the other hand, the trailing component is almost always dominated by one OPM and the respective $V$ distribution favours negative values. Under incoherent addition of OPMs, the observed $V$ is the difference between the $V$ of each OPM and therefore the above observation is in accordance with a particular handedness of $V$ being associated with a certain OPM.
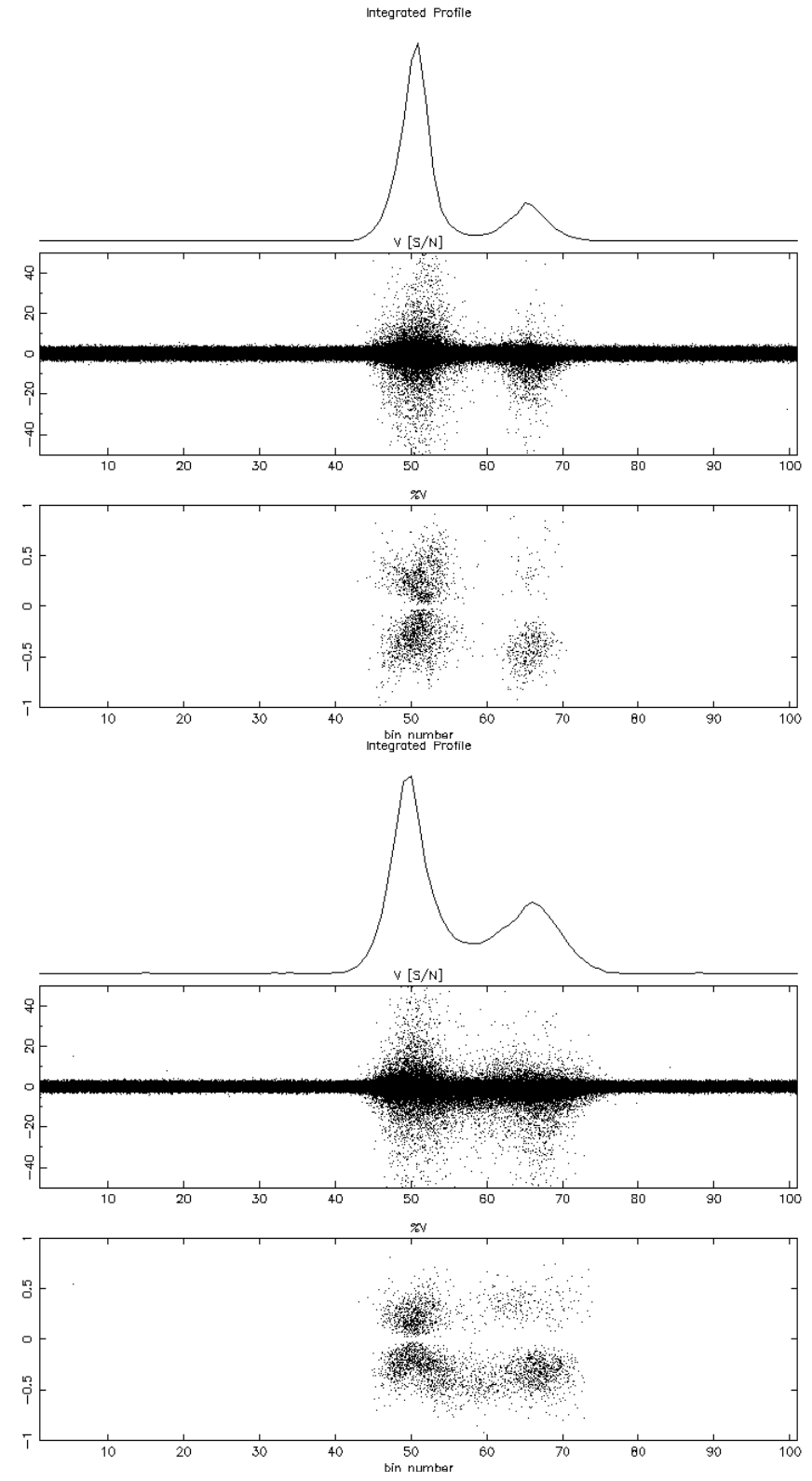

Fig. 1. The distribution of $V$ and $V / I$ in PSR B $1133+16$, at $4.85 \mathrm{GHz}$ (top) and $1.41 \mathrm{GHz}$ (bottom). Each plot has three horizontal panels. The top panel shows the total power $(I)$ integrated pulse profile, the middle panel shows the distribution of flux densities of $V$ in units of the rms and the bottom panel shows the distribution of the fraction $V / I$. In the bottom panel, a threshold has been applied to exclude the noise: only cases where the flux density of $V$ is more than $5 \times \mathrm{rms}$ are shown.

The seemingly bimodal distributions of $V / I$ in the leading component in Fig. 1 are caused by the threshold applied to exclude the noise. Figure 2 shows the specific distributions of $V$ and $V / I$ together with the PA histogram of bin 50. The bottom row corresponds to the $1.41 \mathrm{GHz}$ data and the top row to the $4.85 \mathrm{GHz}$ data. The threshold on $V$ is not applied here, in order to demonstrate how much of the distribution lies within the instrumental noise, the rms of which is represented by a small horizontal bar inside the $V$ distribution. Only the pulses in which the $S / N$ of the total power exceeds 2 and the 

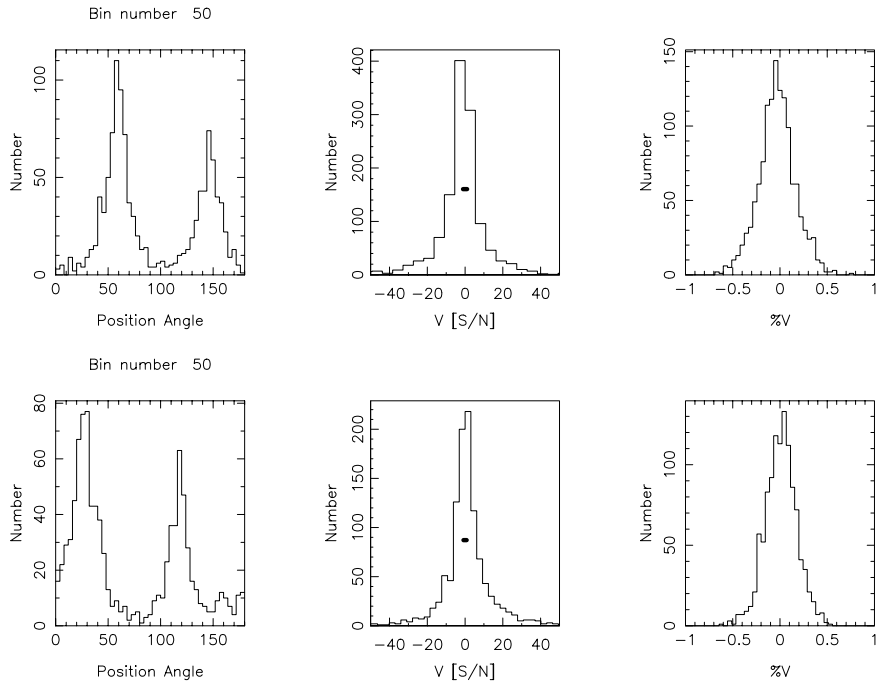

Fig. 2. The distribution of PA, $V$ and $V / I$ in bin 50, near the peak of the leading component, at $4.85 \mathrm{GHz}$ (top) and $1.41 \mathrm{GHz}$ (bottom).
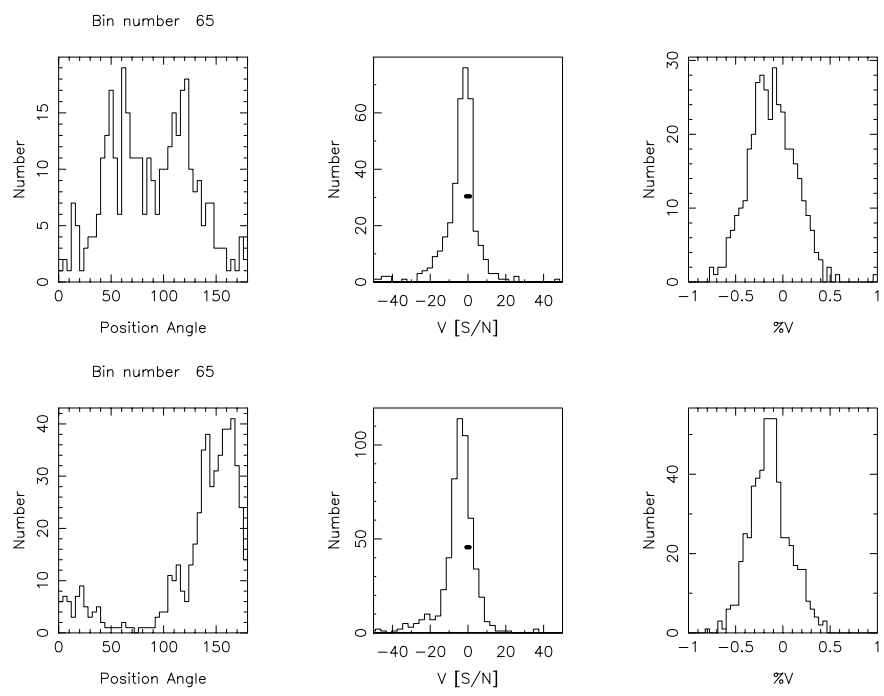

Fig. 3. The distribution of PA, $V$ and $V / I$ in bin 65 , near the peak of the trailing component, at $4.85 \mathrm{GHz}$ (top) and $1.41 \mathrm{GHz}$ (bottom).

fractional linear polarization exceeds $20 \%$ are considered, in order to exclude ambiguous PA values. Bin 50 exhibits a clear bimodal PA distribution at both frequencies and at the same time a mean $V$ value very close to 0 . On the other hand, Fig. 3 shows bin 65 from the trailing pulse component, that presents a slightly different picture. The PA distributions at both frequencies are dominated by one OPM (at $4.85 \mathrm{GHz}$ this is not entirely clear due to the small population of points that survive the threshold) and the $V$ distributions have a significantly nonzero negative mean. This picture is consistent with incoherently superposed OPM, each mode favouring a particular handedness of $V$ : in bin 50 where both modes dominate equally often, $V$ is distributed around 0 , which is not the case for bin 65 , where one mode essentially dominates and the handedness of $V$ associated with this mode gives mean $V$ its negative value.

Figures 2 and 3 show a difference between the distributions in $V$ and $V / I$. All four $V / I$ histograms can be adequately approximated by Gaussian distributions. In bin 50, the best fit has a mean of $-2.5 \%$ and a $\sigma$ of $19 \%$ at $4.85 \mathrm{GHz}$ and a mean of $0.6 \%$ and a $\sigma$ of $18 \%$ at $1.41 \mathrm{GHz}$. In bin 65 on the other hand, the best fit has a mean of $-12 \%$ and a $\sigma$ of $27 \%$ at $4.85 \mathrm{GHz}$ and a mean of $-13 \%$ and a $\sigma$ of $24 \%$ at $1.41 \mathrm{GHz}$. These broad distributions are consistent with those seen in other pulsars (Stinebring et al. 1984a,b) and are largely dominated by instrumental noise (McKinnon 2002).

In summary, the density plots of the circular polarization in Fig. 1 and the individual distributions in Figs. 2 and 3, together with the OPM analysis presented in Paper II, provide evidence that each OPM is associated with a certain handedness of $V$. This association, however, is obviously not perfect, and we discuss this in the following.

\subsection{The $V$-OPM correlation}

In Cordes et al. (1978), observations of PSR B2020+28 at $404 \mathrm{MHz}$ were used to investigate a possible connection between the handedness of $V$ and the PA value. To this goal, they plotted $V$ versus PA in the single pulses they observed. In their paper, they give an example of a particular pulse longitude where the distribution of PAs is bimodal, in other words a phase bin where the dominant OPM alternates from pulse to pulse. The plot of $V$ versus PA for this bin demonstrates a clear correlation between the handedness of $V$ and the OPM.

In Paper II, a detailed study of the frequency dependence of the OPMs in PSR B1133+16 demonstrated that, at higher frequencies, the OPMs are seen to dominate almost equally often and the individual flux densities of the OPMs become more equal to each other. Given this frequency evolution, we investigate whether the $V$-OPM association is also frequency dependent. Instead of $V$-PA plots, we follow a different route, namely to display the tips of the polarization vectors of all the single pulses from specific bins on the Poincare sphere. The coordinates of a given point on the surface of the sphere are given by two angles, the latitude $\left(\tan ^{-1} V / L\right)$ and longitude (2PA). The latitude is a measure of the ellipticity of the polarization state: values of $0^{\circ}$ correspond to linearly polarized states whereas values of $\pm 90^{\circ}$ correspond to purely circular states of opposite handedness. Figure 4 shows the bimodal distributions of PA in phase bin 50, the modes being $\sim 90^{\circ}$ apart in PA. It also shows that the majority of points in each mode prefer a certain handedness in $V$ and therefore lie on either side of the equator $(V=0)$. Comparing Fig. 4 to Fig. 6 in Cordes et al. (1978), reveals that the association of $V$ with PA is not as clear in PSR B1133+16 at these frequencies as it was in PSR B2020+28 at $404 \mathrm{MHz}$. A quantitative test is therefore necessary.

In the scheme of OPM superposition, it is very important to stress again that the observed PA is that of the dominant mode, regardless of the flux density difference of the two modes. Therefore, by testing the PA vs. the sign of $V$ association, one is effectively testing the association of the handedness of $V$ with OPM. For each phase bin of the pulse and for the total number of pulses, we quantize the observed quantities according to the following $3 \times 2$ possibilities: dominant mode 1 , dominant mode 2 or not defined PA, and positive- $V$ 

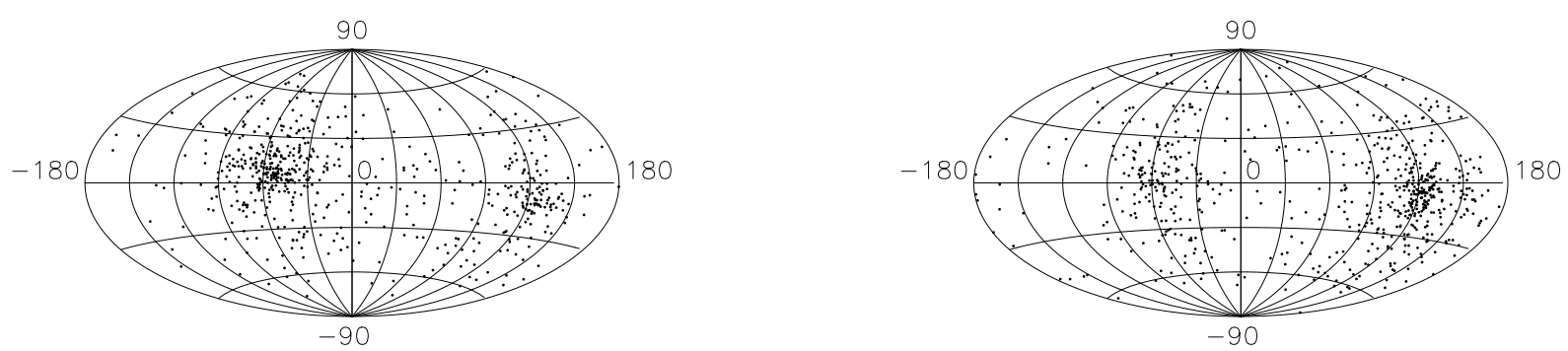

Fig. 4. Hammer-Aitoff projection of the Poincaré sphere, where the polarization states of a phase bin 50 from the single pulses are depicted as the tip of the polarization vector on the Poincaré sphere. The left panel corresponds to $1.41 \mathrm{GHz}$ and the right panel to $4.84 \mathrm{GHz}$. Bimodal PA distributions are evident in both plots.

Table 1. Contingency tables for bin 50 of the PSR B1133+16 data, showing the frequency of occurrence of all possible combinations in the single pulses. The top table corresponds to the $4.85 \mathrm{GHz}$ and the bottom table to the $1.41 \mathrm{GHz}$ data.

\begin{tabular}{c|ccc}
\hline \hline $4.85 \mathrm{GHz}$ & Mode 1 & Mode 2 & Undefined \\
\hline$V+$ & 390 & 452 & 1366 \\
$V-$ & 659 & 436 & 1475 \\
\hline \hline $1.41 \mathrm{GHz}$ & Mode 1 & Mode 2 & Undefined \\
\hline$V+$ & 269 & 632 & 1506 \\
$V-$ & 442 & 386 & 1563 \\
\hline
\end{tabular}

or negative- $V$. By composing such contingency tables for each phase bin, it is possible to quantify the association between the handedness of $V$ and the dominant OPM. An example for bin 50 at each observed frequency is shown in Table 1 . The numbers have been obtained by applying a two step process. Where the $S / N$ of $L$ in the given bin is less than 2.5, we are unable to compute the PA and therefore classify these as "Undefined" in Table 1. Then, we compare the observed PA in each single pulse to the PA of the integrated profile in this bin, to determine which mode is dominating at this particular instant. From the numbers presented in Table 1, it is possible to compare the observed cases to the null hypothesis, by using the formula

$D= \pm \frac{(O b s-N u l l)^{2}}{N u l l}$

where $O b s$ are the observed numbers and Null the null hypothesis. Negative values denote a deficit of the observed cases from the null hypothesis. For every bin such as the one presented in Table 1 there will be four values of $D$, one for each table element.

We perform this analysis across the whole profile of PSR B1133+16. What we find is that there is an excess from the null hypothesis of negative $V$ cases across the pulse for the most common OPM (Mode 1) and a positive $V$ excess from the null hypothesis for the other OPM (Mode 2). Figure 5 shows $D$ as measured by Eq. (1), plotted across the pulse. The top panel of the plot shows the $D$ values for the association between Mode 1 and negative $V$, whereas the bottom panel shows the same for Mode 2 and positive $V$, at both frequencies. From the definition of $D$ we find that values over 5 are statistically significant. Two facts are evident from these plots. First, the values of $D$ are generally large enough to support a strong correlation between the handedness of $V$ and the dominant

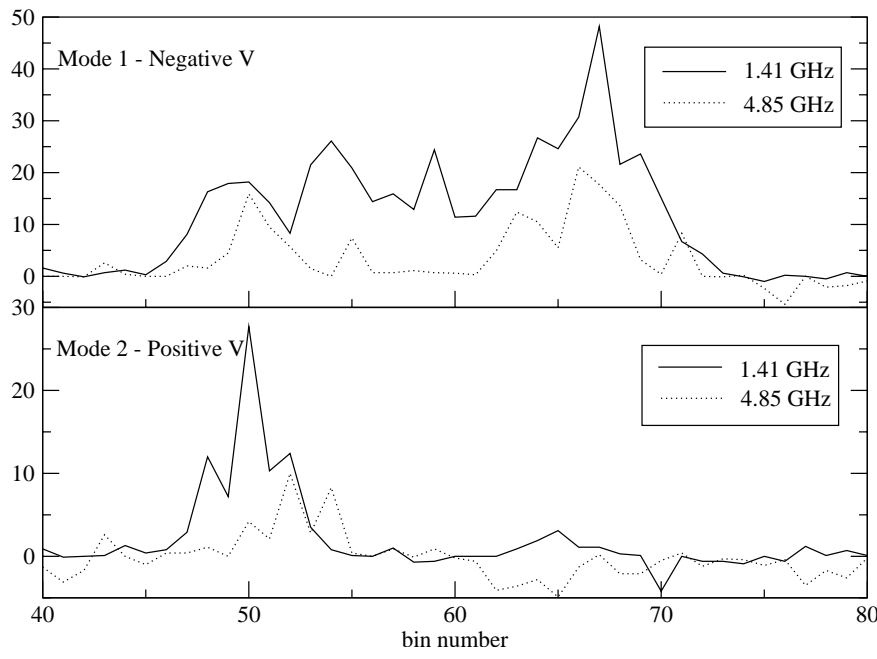

Fig. 5. $D$ plotted against the pulse phase bin for the Mode 1-Vand Mode $2-V+$ cases. The solid line corresponds to $1.41 \mathrm{GHz}$ and the dotted line to $4.85 \mathrm{GHz}$. The negative $D$ values signify a deficit from the null hypothesis.

OPM. Second, the $D$ values are consistently larger at $1.41 \mathrm{GHz}$ than at $4.85 \mathrm{GHz}$, indicating that the correlation is weaker at $4.85 \mathrm{GHz}$. Also, as we have seen before, Mode 2 hardly ever dominates in the trailing pulse component and therefore the corresponding $D$ values are all close to zero.

\section{OPM separation}

Our statistical analysis of the dual frequency PSR B1133+16 data has shown us that there is a frequency dependent correlation between the handedness of $V$ and the dominant OPM. The contingency tables, however, show that there is a significant population of cases where the handedness of $V$ is opposite from the expected. In other words, the $V$-OPM association is not perfect. This is a very important result with serious implications on the technique of decomposing pulse profiles into the individual OPMs. The significance lies in the fact that each OPM that dominates in a given single pulse may be observed instantaneously with either handedness of $V$. However, averaging the single pulses and decomposing the average profile is based on an assumption that is not met, namely that each OPM is clearly associated with a particular sign of $V$ in all the single pulses. To demonstrate this, we detail the mathematics of mode-decomposition in single pulses and show the difference 

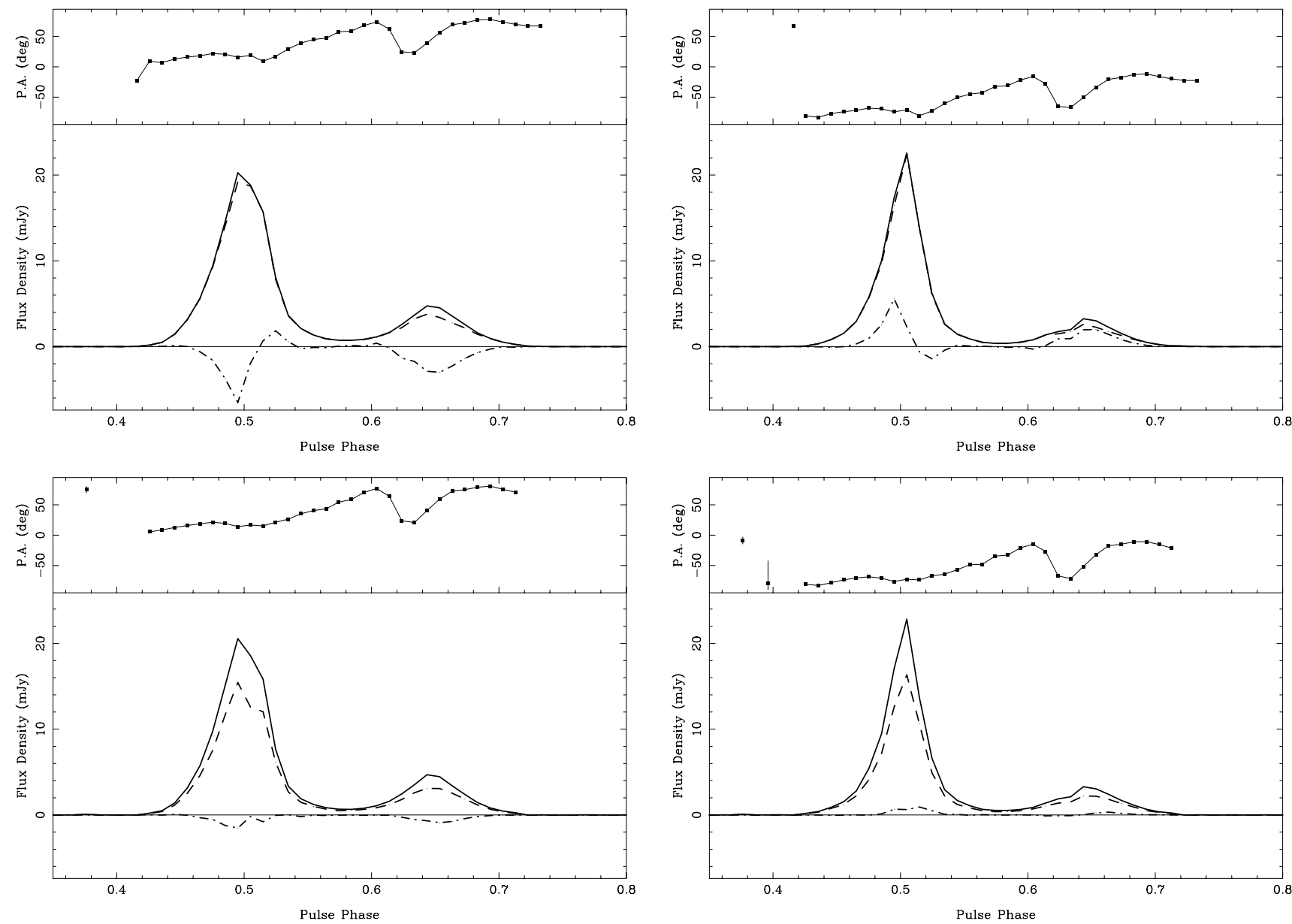

Fig. 6. The top row of plots shows the average pulse profile of PSR $1133+16$ at $4.85 \mathrm{GHz}$, decomposed into the profiles for Mode 1 (left) and Mode 2 (right). The bottom row is the result of the decomposition into the individual OPMs of each single pulse, which are then averaged. In each plot, the main panel shows the total power $I$ (solid), the linear polarization $L$ (dashed) and the circular polarization $V$ (dashed-dotted). The secondary panel on top shows the PA.

between the obtained profiles by decomposing and then averaging, and averaging and then decomposing the PSR B1133+16 data.

The orthogonality of the modes means that their polarization states at a given instant are represented by antiparallel vectors on the Poincaré sphere. By definition therefore the PAs can only take values which are exactly $90^{\circ}$ apart. Incoherent superposition allows the addition of the individual Stokes parameters. The mathematical process is as follows:

The observed Stokes parameters can be written in terms of the OPMs

$I=I_{1}+I_{2}$

$Q=Q_{1}+Q_{2}$

$U=U_{1}+U_{2}$

$V=V_{1}+V_{2}$

$L=L_{1}+L_{2}$,

where $L_{i}=\sqrt{Q_{i}^{2}+U_{i}^{2}}$. The $100 \%$ polarized modes add up to produce the observed, partially polarized radiation, where $L^{2}+V^{2} \neq I^{2}$. Defining $P$ as the polarized intensity $\sqrt{L^{2}+V^{2}}$, and considering that the antiparallel vectors must have opposite senses of circular polarization, the orthogonal polarization states imply that,

$\frac{L_{i}}{I_{i}}=d_{L}=\frac{L}{P}$,
$\frac{\left|V_{i}\right|}{I_{i}}=d_{V}=\frac{V}{P}$.

Also, the OPMs are $100 \%$ polarized, which means that

$d_{L}^{2}+d_{V}^{2}=1$ or $d_{V}=\sqrt{1-d_{L}^{2}}$.

Taking into account the antiparallel vectors of the OPM polarization states and ignoring $Q$ and $U$ for the moment, Eq. (2) can be written as

$I=I_{1}+I_{2}$

$L=d_{L}\left(I_{1}-I_{2}\right)$

$V=\sqrt{1-d_{L}^{2}}\left(I_{1}-I_{2}\right)$,

which are sufficient to solve for $I_{1}$ and $I_{2}$, so that

$I_{1}=0.5\left(I+\frac{L}{d_{L}}\right)$

$I_{2}=0.5\left(I-\frac{L}{d_{L}}\right)$, 

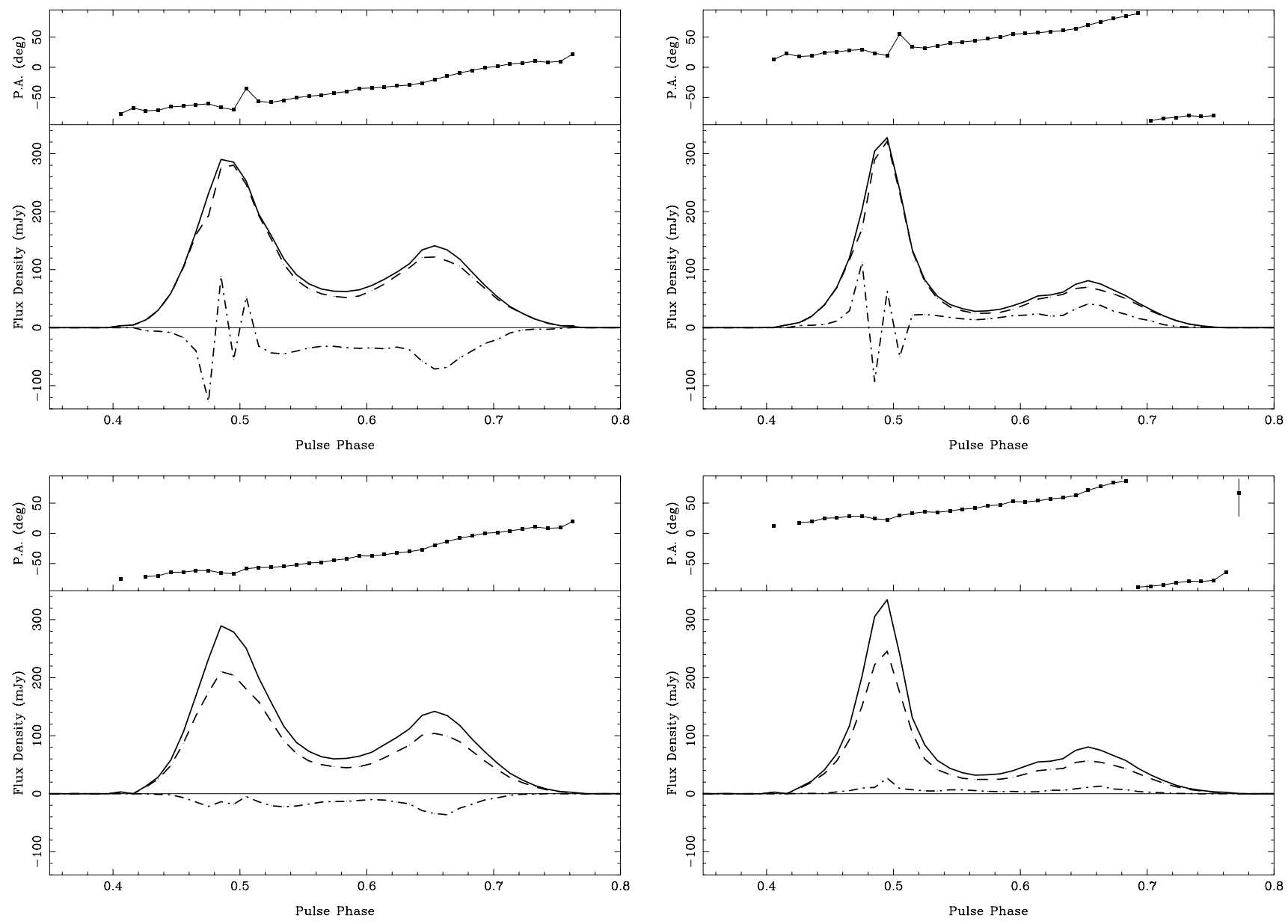

Fig. 7. Same as Fig. 6 for $1.41 \mathrm{GHz}$.

and therefore, Eqs. (3) and (4) lead to

$$
\begin{aligned}
& L_{1}=d_{L} \cdot I_{1} \\
& L_{2}=d_{L} \cdot I_{2} \\
& V_{1}=d_{V} \cdot I_{1} \\
& V_{2}=-d_{V} \cdot I_{2} .
\end{aligned}
$$

To decompose $Q$ and $U$, the PA is used. The PA of the strongest mode should be equal to the observed PA, and the PA of the weak mode should be orthogonal to this. Therefore,

$\frac{U}{Q}=\frac{U_{1}}{Q_{1}}=\frac{-U_{2}}{-Q_{2}}=\frac{U_{2}}{Q_{2}}$,

from which $Q_{i}$ and $U_{i}$ can be determined as

$$
\begin{aligned}
Q_{i} & =\frac{L_{i}}{L} \cdot Q \\
U_{i} & =\frac{L_{i}}{L} \cdot U .
\end{aligned}
$$

The above method shows that there is a unique way to decompose the received Stokes parameters into the Stokes parameters of the individual orthogonal modes. In practice, special care must be taken in the cases of very small $L$ and $V$ to avoid significant errors. The equations also express the intrinsic assumption of this method that the modes are orthogonal, so the circular polarization sense is clearly opposite in each mode in a given single pulse. However, the sense of $V$ that is associated with a particular OPM may change from pulse to pulse.

In Stinebring \& McKinnon (2000), the average profiles of PSRs B0525+21 and B2020+28 are decomposed into the OPMs, using the assumption of superposed OPM emission. We apply our method of single-pulse decomposition and their method of average profile decomposition to PSR B1133+16 to identify and interpret the differences. Figures 6 and 7 show the results of the two different approaches to OPM-decomposition at $4.85 \mathrm{GHz}$ and $1.41 \mathrm{GHz}$ respectively. First of all, the averaged profiles created using the two methods are obviously different. The average circular polarization in the case of the single-pulse decomposition has a constant sense across the profile, which is not true for the average profile decomposition. Also, it can be seen that the average flux density of $V$ in each of the modes is considerably smaller in the case of single-pulse decomposition. Another aspect of the observed differences between the profiles is the linear polarization, which is $\sim 100 \%$ in the decomposed average profiles and significantly less in the decomposed single-pulse profiles. We claim that the reduced degree of polarization is due to the spread in the PA distributions. We have found that a $45^{\circ}$ spread in the PA distribution of a particular phase bin, results in a $\sim 20 \%$ decrease in the mean linear polarization after averaging a few hundreds of 

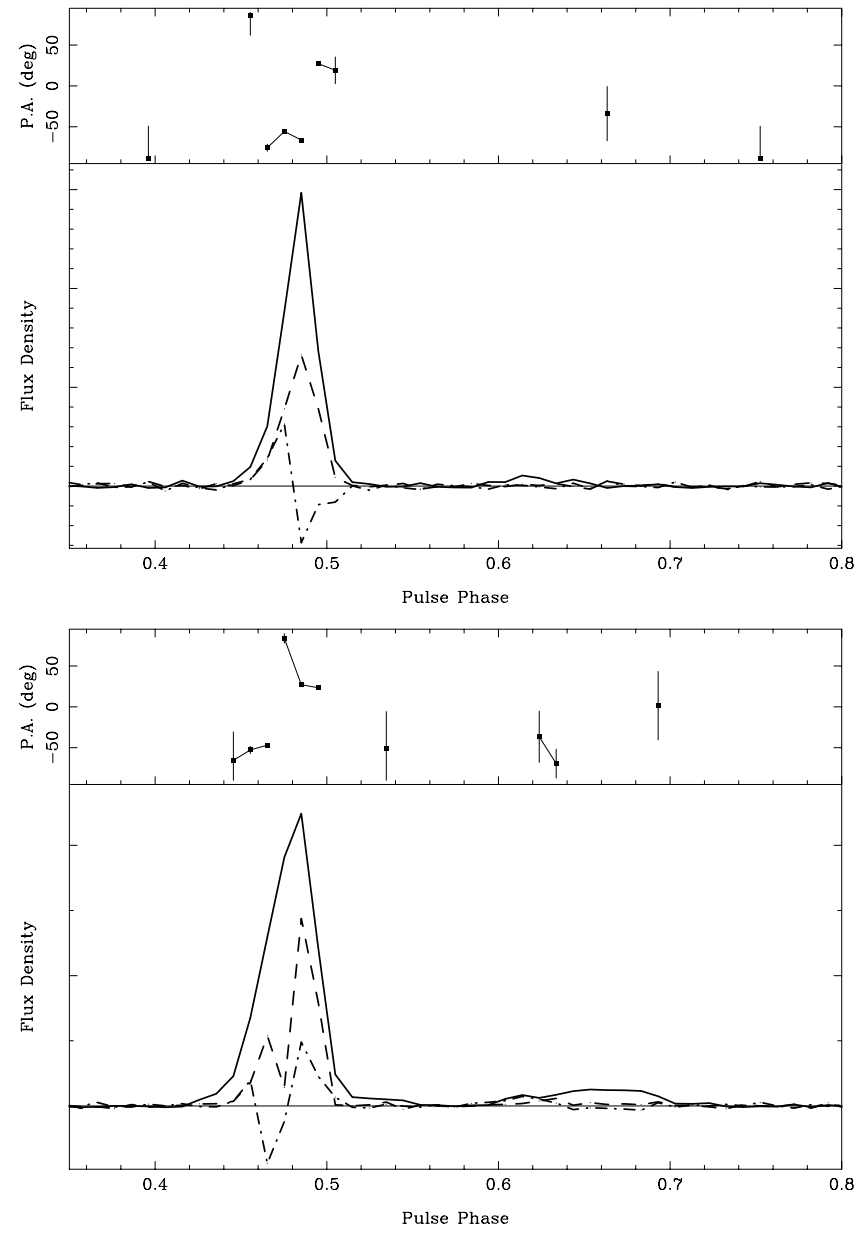

Fig. 8. Single pulse number 1562 from PSR B1133+16, at $4.85 \mathrm{GHz}$ (top) and $1.41 \mathrm{GHz}$ (bottom). The solid line represents the total power, the dashed line the linear polarization, and the dashed-dotted line the circular polarization. The PA jumps in the same direction at both frequencies, but the circular polarization follows a swing of opposite handedness between the frequencies.

single pulses. For this reason, it is impossible to obtain modeseparated average profiles constructed from decomposed single pulses that exhibit $100 \%$ polarization unless the PA distributions are delta-functions.

\section{Simultaneous pulses}

The significant correlation between $V$ and OPM is in agreement with the initial result for PSR B2020+28 (Cordes et al. 1978) and constitutes evidence that circular polarization is closely linked to the OPM phenomenon. However, we have demonstrated that decomposing single pulses into the OPMs and averaging produces different mode-separated profiles from averaging single pulses first and then decomposing the resulting profile. This happens because the correlation of $V$ with OPM, although significant, is not perfect, and becomes worse with frequency. Obviously, the entire mechanism that determines the polarization we observe is not well understood.

In Karastergiou et al. (2000, Paper I) and Paper II it was shown that single pulses of PSRs B1133+16 and B0329+54 observed simultaneously at two, widely spaced frequencies
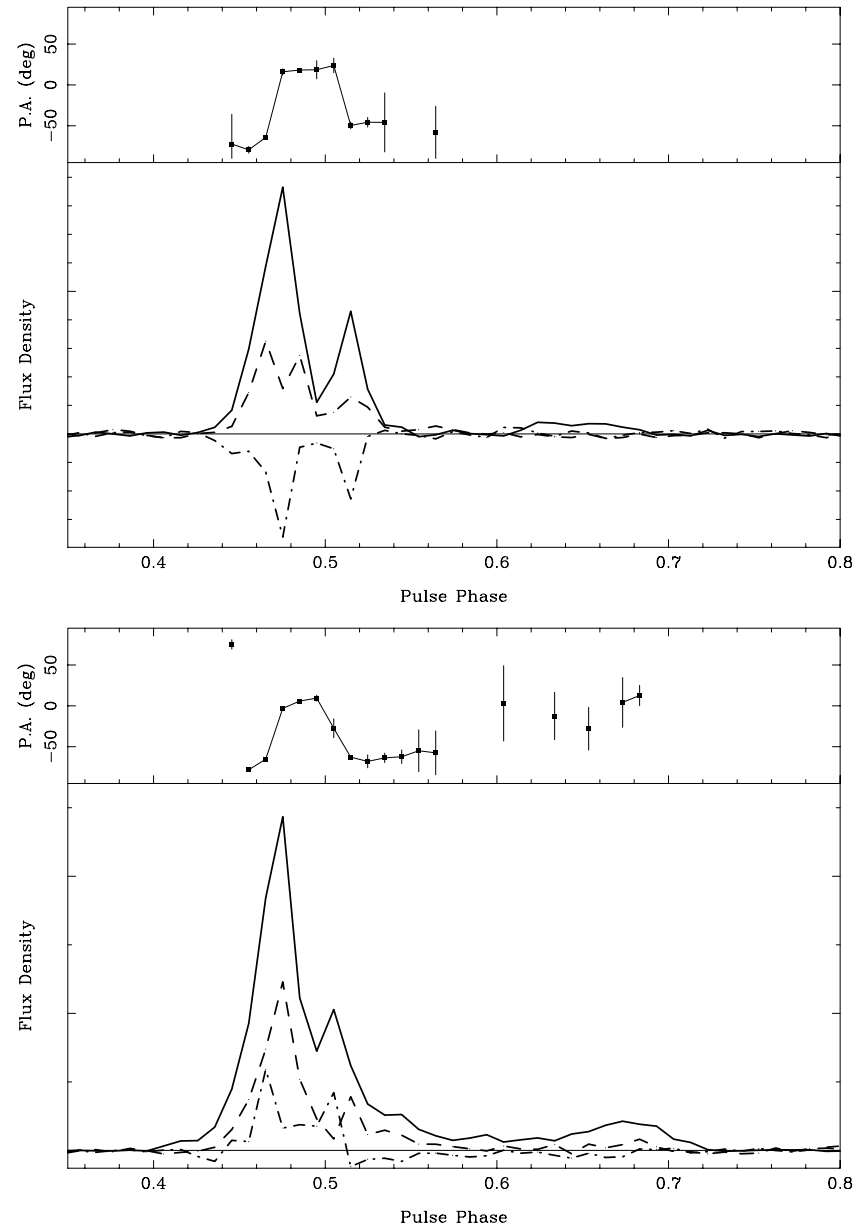

Fig. 9. Single pulse number 2438 from PSR B1133+16, at $4.85 \mathrm{GHz}$ (top) and $1.41 \mathrm{GHz}$ (bottom). The circular polarization disagrees in handedness between the frequencies. The $V$ flux densities match very well, showing a peak at each phase bin of the PA jump.

may often disagree in terms of the dominant OPM. Despite these frequent cases of disagreement, the cases of agreement were statistically more significant and therefore revealed a high degree of correlation in OPMs between the observed frequencies. The correlation of OPMs between frequencies is facilitated by the fact that the PAs of the OPMs are, by definition, orthogonal. Therefore, it is possible to distinguish the prevailing OPM at both frequencies and count the cases of agreement and disagreement. Unfortunately, the observed $V$ has wide unimodal distributions around a usually very small mean. An investigation of the correlation of the handedness of $V$ between the frequencies, is therefore dominated by the mean and the rms of the $V$ distribution. As an example, the bins in the trailing pulse component of PSR B1133+16 as seen in Fig. 1, show broad $V$ distributions of a clearly negative mean. A test of the association in handedness of $V$ between the frequencies in this component obviously shows a high correlation, forced by the underlying distributions themselves. As for the leading pulse component, we have demonstrated that each mode is clearly associated to a particular handedness in $V$ at a given frequency. The high degree of the correlation of the OPMs between the frequencies therefore also results in a high correlation in the 
handedness of $V$. To summarize, we see a high correlation in the handedness of $V$ between the observed frequencies, which is what we expect from our previous knowledge. This correlation, however, does not add significantly to our interpretation of the observations.

Given the simultaneity of our data, we have the opportunity to inspect single pulses as a whole observed at both frequencies. Two example single-pulses are provided in Figs. 8 and 9. Figure 8 shows pulse number 1562 from PSR B1133+16 at $4.85 \mathrm{GHz}$ (top panel) and $1.41 \mathrm{GHz}$ (bottom panel). The leading pulse component shows the characteristic swing and change of handedness in $V$ usually associated with core components. The sense of the swing is opposite between the two frequencies although the PAs agree. They both show a PA jump in the same direction, although the jump happens slightly earlier at the lower frequency. In pulse 2438 in Fig. 9, an OPM jump occurs in bin 48 and the PA jumps back again in bin 52, at both frequencies. Both jumps correspond closely to the peaks in $V$. The shape of the $V$ profile around these bins is also very similar between the two frequencies, but the handedness is opposite. The examples also help to extend the significance of our results to timescales comparable to the pulsar component widths.

\section{Summary}

In this paper we have demonstrated a method for decomposing single pulses into the OPMs, assuming incoherently superposed OPM emission. We support this method in light of our findings for PSR B1133+16, where the handedness of the circular polarization has been observed not to be perfectly associated to the dominant OPM. We have shown that the correlation of $V$ with OPM is worse at $4.85 \mathrm{GHz}$ than at $1.41 \mathrm{GHz}$, which is consistent with the conclusion of Paper II that propagation through the pulsar magnetosphere causes more severe effects at higher rather than lower frequencies. Finally, we show two examples of single pulses to demonstrate that our bin-bybin analysis is also applicable to structures with timescales similar to pulsar components.

Acknowledgements. The authors would like to thank everybody at the Effelsberg and Lovell radio telescopes who helped in any way with the observations. AK would like to thank the Deutscher Akademischer Austausch Dienst for their financial support.

\section{References}

Barnard, J. J., \& Arons, J. 1986, ApJ, 302, 138

Cordes, J. M., Rankin, J. M., \& Backer, D. C. 1978, ApJ, 223, 961

Karastergiou, A., Kramer, M., Johnston, S., et al. 2002, A\&A, 391, 247

Karastergiou, A., von Hoensbroech, A., Kramer, M., et al. 2001, A\&A, 379,270

McKinnon, M. 1997, ApJ, 475, 763

McKinnon, M., \& Stinebring, D. 1998, ApJ, 502, 883

McKinnon, M. M., \& Stinebring, D. R. 2000, ApJ, 529, 435

Petrova, S. A. 2001, A\&A, 378, 883

Stinebring, D. R., Cordes, J. M., Rankin, J. M., Weisberg, J. M., \& Boriakoff, V. 1984a, ApJS, 55, 247

Stinebring, D. R., Cordes, J. M., Weisberg, J. M., Rankin, J. M., \& Boriakoff, V. 1984b, ApJS, 55, 279

von Hoensbroech, A., Lesch, H., \& Kunzl, T. 1998, A\&A, 336, 209 